OPEN ACCESS

Edited by:

Ichiro Abe,

Fukuoka University Chikushi Hospital, Japan

Reviewed by: Michio Otsuki,

Osaka University Hospital, Japan Wataru Kameda,

Yamagata University, Japan

*Correspondence:

Hironobu Sasano

hsasano@patholo2.med.tohoku.ac.jp

Specialty section:

This article was submitted to Neuroendocrine Science,

a section of the journal

Frontiers in Endocrinology

Received: 27 July 2020 Accepted: 09 October 2020 Published: 10 November 2020

Citation:

Gao X, Yamazaki Y, Pecori A Tezuka Y, Ono Y, Omata $K$, Morimoto R, Nakamura $Y$, Satoh F and Sasano H (2020) Histopathological Analysis of

Tumor Microenvironment and Angiogenesis in Pheochromocytoma.

Front. Endocrinol. 11:587779. doi: 10.3389/fendo.2020.587779

\section{Histopathological Analysis of Tumor Microenvironment and Angiogenesis in Pheochromocytoma}

\author{
Xin Gao ${ }^{1}$, Yuto Yamazaki ${ }^{1}$, Alessio Pecori ${ }^{1}$, Yuta Tezuka ${ }^{2,3}$, Yoshikiyo Ono ${ }^{3}$, \\ Kei Omata ${ }^{2,3}$, Ryo Morimoto ${ }^{3}$, Yasuhiro Nakamura ${ }^{4}$, Fumitoshi Satoh ${ }^{2,3}$ \\ and Hironobu Sasano ${ }^{1 *}$ \\ ${ }^{1}$ Department of Pathology, Tohoku University Graduate School of Medicine, Sendai, Japan, ${ }^{2}$ Division of Clinical \\ Hypertension, Endocrinology and Metabolism, Tohoku University Graduate School of Medicine, Sendai, Japan, ${ }^{3}$ Division of \\ Nephrology, Endocrinology, and Vascular Medicine, Tohoku University Hospital, Sendai, Japan, ${ }^{4}$ Division of Pathology, \\ Faculty of Medicine, Tohoku Medical and Pharmaceutical University, Sendai, Japan
}

Pheochromocytomas (PHEOs) are relatively rare catecholamine-producing tumors derived from adrenal medulla. Tumor microenvironment (TME) including neoangiogenesis has been explored in many human neoplasms but not necessarily in PHEOs. Therefore, in this study, we examined tumor infiltrating lymphocytes (CD4 and CD8), tumor associated macrophages (CD68 and CD163), sustentacular cells (S100p), and angiogenic markers (CD31 and areas of intratumoral hemorrhage) in 39 cases of PHEOs in the quantitative fashion. We then compared the results with pheochromocytoma of the adrenal gland scaled score (PASS), grading system for pheochromocytoma and paraganglioma (GAPP) and the status of intra-tumoral catecholamine-synthesizing enzymes (TH, DDC, and PNMT) as well as their clinicopathological factors. Intratumoral CD8 ( $p=0.0256)$, CD31 ( $p=$ $0.0400)$, and PNMT $(p=0.0498)$ status was significantly higher in PHEOs with PASS $<4$ than PASS $\geqq 4$. In addition, intratumoral $\mathrm{CD}^{+}$Iymphocytes were also significantly more abundant in well-than moderately differentiated PHEO according to GAPP score ( $\mathrm{p}=$ $0.0108)$ and inversely correlated with tumor size $(p=0.0257)$. Intratumoral $\mathrm{CD} 68^{+}$cells were significantly higher in PHEOs with regular or normal histological patterns than those not ( $p=$ $0.0370)$ and inversely correlated with tumor size $(p=0.0457)$. The status of CD163 was significantly positively correlated with that of CD8 positive cells ( $p=0.0032)$. The proportion of intratumoral hemorrhage areas was significantly higher in PHEOs with PASS $\geqq 4$ ( $p=$ 0.0172). DDC immunoreactivity in tumor cells was significantly positively correlated with PASS score $(p=0.0356)$ and TH status was significantly higher in PHEOs harboring normal histological patterns $(p=0.0236)$ and cellular monotony $(p=0.0219)$ than those not. Results of our present study did demonstrate that abundant $C D 8^{+}$and $C D 68^{+}$cells could represent a histologically low-scored tumor. In particular, PHEOs with increased intratumoral hemorrhage should be considered rather malignant. In addition, abnormal catecholamine-producing status of tumor cells such as deficient PNMT and $\mathrm{TH}$ and increased DDC could also represent more aggressive PHEOs.

Keywords: pheochromocytoma, pathology, immunohistochemistry, tumor microenvironment, angiogenesis 


\section{INTRODUCTION}

Pheochromocytomas (PHEOs) and paragangliomas (PGLs) are relatively rare tumors originating from the adrenal medulla at proximately 2 to 9.1 per 1 million adults frequently associated with cardiovascular complications due to excessive catecholamine production (1-3). The difference between PHEOs and PGLs depends on the primary sites, i.e., intra- or extra-adrenal glands (4), and the former one termed as PHEOs (5). It is generally extremely difficult to differentiate benign from malignant PHEOs based upon clinical or even histopathological findings, and all PHEOs are currently considered potentially malignant tumor in the WHO 2017 classification (6). However, toward establishing more accurate histopathological diagnosis of PHEOs, Thompson et al., proposed a novel scoring system for the patients with PHEOs, i.e., PASS (pheochromocytoma of the adrenal gland scaled score) system, composed of twelve different histological features (7). This system has been frequently used for discerning malignancy in PHEOs, and PASS of $\geqq 4$ was proposed to be malignant or biologically more aggressive than those with PASS <4 (7). However, it is also true that there have been controversies regarding the application of this system into the differential diagnosis between benign and malignant PHEOs including its reproducibility (7). Kimura et al. subsequently proposed another system to access malignant potential of PHEOs, i.e., GAPP (grading system for pheochromocytoma and paraganglioma) (8). This system used both histological and clinical parameters including histological patterns, cellularity, comedo-type necrosis, capsular/ vascular invasion, proliferative index (Ki-67) and catecholamine type. According to the GAPP system, PEHOs were subclassified into well-differentiated (WD), moderately differentiated (MD), or poorly differentiated tumor (PD), and this classification has been then used widely.

The tumor microenvironment (TME) has been reported to play pivotal roles in many human malignancies in tumorigenesis, progression, and metastasis and recently also explored as therapeutic targets (9). Histopathologically, TME is well known to be composed of a number of cellular components including inflammatory cells, blood vessels, fibroblasts, and others $(10,11)$. Of those components above, both T lymphocytes and macrophages have been generally considered to play pivotal roles in biological features of the patients $(12,13)$. In benign adrenocortical tumors, we previously demonstrated that cortisol-producing adenomas harbored higher immune cell infiltration and angiogenic markers than other hormone-producing adenomas (14). In adrenocortical carcinomas, tumor infiltrating $\mathrm{T}$ cells were also reported to be correlated with better overall survival (15). However, little has been known on TME in PHEOs at this juncture even in contrast to adrenocortical neoplasms above, although aggressive PHEOs were reported to harbor lower number of S100 positive sustentacular cells and increased angiogenesis (16-18). Therefore, in this study, we explored various TME relevant markers (CD4, CD8, CD68, CD163, and S100p), angiogenic markers (CD31, intratumoral hemorrhage area) and catecholamine-synthesizing enzymes (TH, DDC, and PNMT) of the tumors and compared the results with GAPP and PASS scoring systems, other clinicopathological factors of individual cases of 39 PHEOs in order to explore the possible correlation between the status of catecholamine production and TME and angiogenesis.

\section{MATERIALS AND METHODS}

\section{Pheochromocytoma Cases}

We studied 39 adrenal PHEO patients operated at Tohoku University Hospital, Sendai, Japan from 2012 to 2019. Following the evaluation of $24 \mathrm{~h}$ urinary levels of metanephrine and normetanephrine, the diagnosis of PHEO was confirmed by their increased levels of at least three times than the normal range (2). We also applied PASS and GAPP in their histological diagnosis. Clinicopathological features of these patients examined were summarized in Table 1. Clinical information is not available due to the relatively new cases. Two patients experienced recurrence and one of them died. The present research protocol was approved by the Institutional Review Board (IRB) of Tohoku University School of Medicine (2018-1-669).

\section{Immunohistochemistry and Its Evaluation}

Hematoxylin and eosin (H\&E) staining and immunohistochemistry (IHC) were both performed in the specimens fixed in $10 \%$ buffered formalin and embedded in paraffin. The protocols for individual IHC markers used in our present study were summarized in Table 2. All the H\&E and IHC sections were digitally scanned by Image Scope AT2 (Leica, Wetzler, Germany). Nuclear immunoreactivity of CD4, CD8, CD68, CD163, and S100p were all evaluated by the percentage of positive cells in tumoral parenchyma by manual analysis (19-22). Ki-67 labeling index was evaluated after identifying hot spot of the whole tumor (23). Microvascular density (MVD) was evaluated by counting the number of CD31positive vessels within $0.75 \mathrm{~mm}^{2}$ and highest expressed tumor area (24). Immunoreactivity of catecholamine-synthesizing enzymes including tyrosine hydroxylase (TH), dopa-decarboxylase (DDC), and phentolamine-N-methyltransferase (PNMT) was all digitally and quantitatively evaluated by using "HALO" CytoNuclear ver.1.5” (Indica Laboratories, Corrales, NM, USA) image analysis $(25,26)$. The positive cells were tentatively classified into the

TABLE 1 | Clinicopathological factors in PHEO patients.

\begin{tabular}{lc} 
Clinical factors & Average (range) \\
\hline Age (years) & $56(39-79)$ \\
Gender: Female/Gender & $25 / 14$ \\
BMl $\left(\mathrm{kg} / \mathrm{m}^{2}\right)$ & $22.7(15.48-31.49)$ \\
Tumor size $(\mathrm{mm})$ & $45.3(20-100)$ \\
Tumor location: left/right & $24 / 15$ \\
Duration of hypertension (years) & $9.3(0-25)$ \\
Plasma AD (ng/ml) & $0.16(0.01-0.742)$ \\
Plasma NAD (ng/ml) & $1.8(0.1-12)$ \\
24-h urine MN (mg/day) & $1.7(0.01-11)$ \\
24-h urine NMN (mg/day) & $4.1(0.01-26)$ \\
24-h urine AD ( $\mu$ g/day) & $115.7(1.9-510)$ \\
24-h urine NAD ( $\mu \mathrm{gg} /$ day) & $775.9(42.6-4200)$
\end{tabular}

Gender and tumor location were described as the number of persons.

$A D$, adrenaline; $N A D$, noradrenaline; $D A$, dopamine; $M N$, metanephrine; $N M N$, normetanephrine; BMI, body mass index.

Normal range reference: plasma AD: 0-0.17 ng/ml; plasma NAD, $0.15-0.57 \mathrm{ng} / \mathrm{ml} ; 24-\mathrm{h}$ urine adrenaline, 1.1-22.5 mcg/day; 24-h urine noradrenaline, 29.2-118.0 mcg/day; 24- $h$ urine metanephrine, 0.05-0.20 mg/day; 24-h urine normetanephrine, 0.10-0.28 mg/day. 
TABLE 2 | $\mid \mathrm{HC}$ protocols.

\begin{tabular}{lccccc}
\hline Antibody & Host & Clone & Dilution & $\begin{array}{c}\text { Antigen } \\
\text { retrieval }\end{array}$ & Source \\
\hline CD4 & Rabbit & EPR6855 & $1: 400$ & AC & Abcam \\
CD8 & Mouse & M7103 & $1: 50$ & AC & Dako \\
CD68 & Mouse & M0876 & $1: 100$ & Trypsin & Dako \\
CD163 & Mouse & M3527 & $1: 600$ & AC & Dako \\
S100p & Rabbit & - & $1: 9,000$ & - & Dako \\
CD31 & Mouse & M0823 & $1: 100$ & AC & Dako \\
Ki67 & Mouse & M7240 & $1: 100$ & AC & Dako \\
TH & Mouse & T1299 & $1: 2,000$ & MW & Sigma \\
DDC & Rabbit & AB136 & $1: 500$ & - & Millipore \\
PNMT & Mouse & Ab119784 & $1: 2,000$ & - & Abcam \\
\hline AC & & & & &
\end{tabular}

$A C$, autoclave $121^{\circ} \mathrm{C} 5 \mathrm{~min}, \mathrm{pH}=6$ buffer; $\mathrm{MW}$, microwave $500 \mathrm{~W} 20 \mathrm{~min}, \mathrm{pH}=6$ buffer.

following four categories based on the levels of their relative immunointensity: negative as " 0 ", weak as " +1 ", moderate as “ +2 ", and strong as “ +3 ". H-score was subsequently calculated based on the following formula; $\Sigma$ (Number of the individual gradients of the positive cells $\mathrm{X}$ Score $1+, 2+, 3+)$ /Total cells) X100 (27-29). The evaluation of intratumoral hemorrhage area was also performed by using HALO software above according to the classifier system on H\&E stained tissue sections (25). Under this system, tumor areas were tentatively classified into two portions: tumor and intratumoral hemorrhage areas (Figure 1). Hemorrhage in the central vein and ubiquitous necrosis were both carefully excluded in this analysis. The final value of intratumoral hemorrhage was the ratio of intratumoral hemorrhage against the whole tumor area.

\section{Statistical Analysis}

We analyzed the correlations among TME relevant markers, angiogenic markers, catecholamine-synthesizing enzymes, histopathological score, and clinicopathological factors by Spearman's test. The differences of TME relevant markers, angiogenic markers, and catecholamine-synthesizing enzymes in individual PASS and GAPP factors were all analyzed using MannWhitney's test. We defined the significance as P-value $<0.05$. All the tests were analyzed using the software "JMP Pro ver. 14.0.0".

\section{RESULTS}

\section{Comparison of PHEOs Between PASS $\geqq 4$ and PASS $<4$}

Representative images were illustrated in Figure 2. Results were summarized in Figure 3. CD31-positive vessels $(p=0.0400)$, CD8 positive cells $(\mathrm{p}=0.0256), \mathrm{S} 100 \mathrm{p}$ positive cells $(\mathrm{p}=0.0353)$, and PNMT immunoreactivity $(\mathrm{p}=0.0498)$ were all significantly higher in PHEOs with PASS $<4$ than those with PASS $\geqq 4$. The
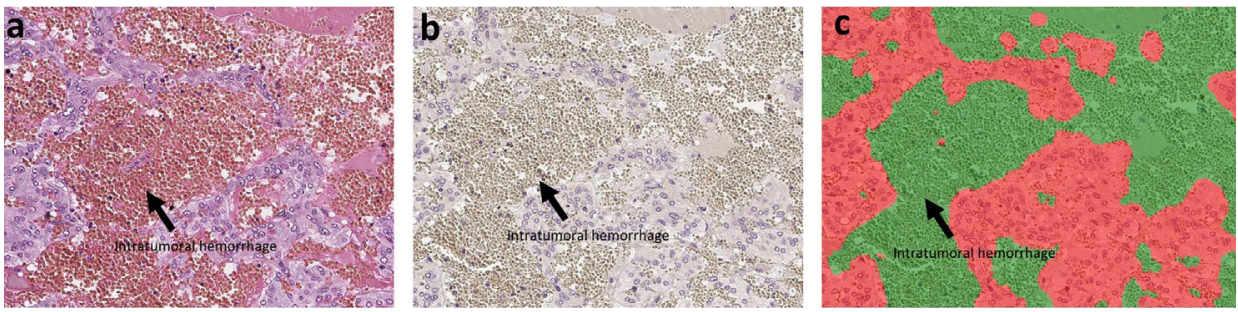

FIGURE 1 | The analysis of intratumoral hemorrhage area. H\&E stained section and hematoxylin-stained section are present in (A, B). There were several intratumoral hemorrhage areas with gray color in (B). Because the hemorrhage is composed of red blood cells that did not have a nucleus, it cannot be stained by hematoxylin. We used classifier system in HALO software to recognize the normal tumor area (blue) and the intratumoral hemorrhage area (gray). After analysis, the software can divide the tissue into two colors: red for tumor and green for hemorrhage (C). Finally, we can calculate the ratio of intratumoral hemorrhage area against the whole tumor area.

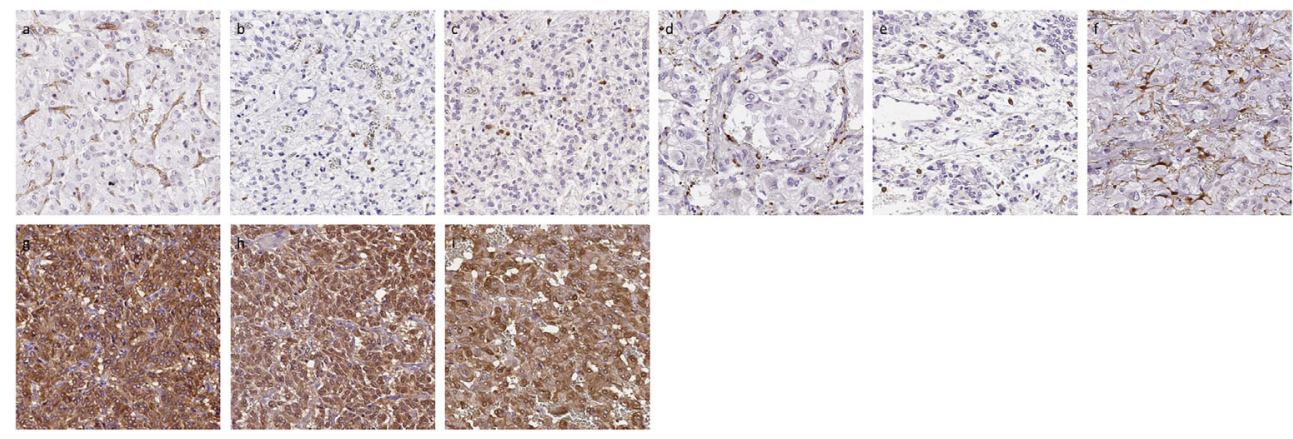

FIGURE 2 | Representative IHC images of PHEOs. (A) CD31, (B) CD4, (C) CD8, (D) CD68, (E) CD163, (F) S100p, (G) TH, (H) DDC, (I) PNMT. 
A
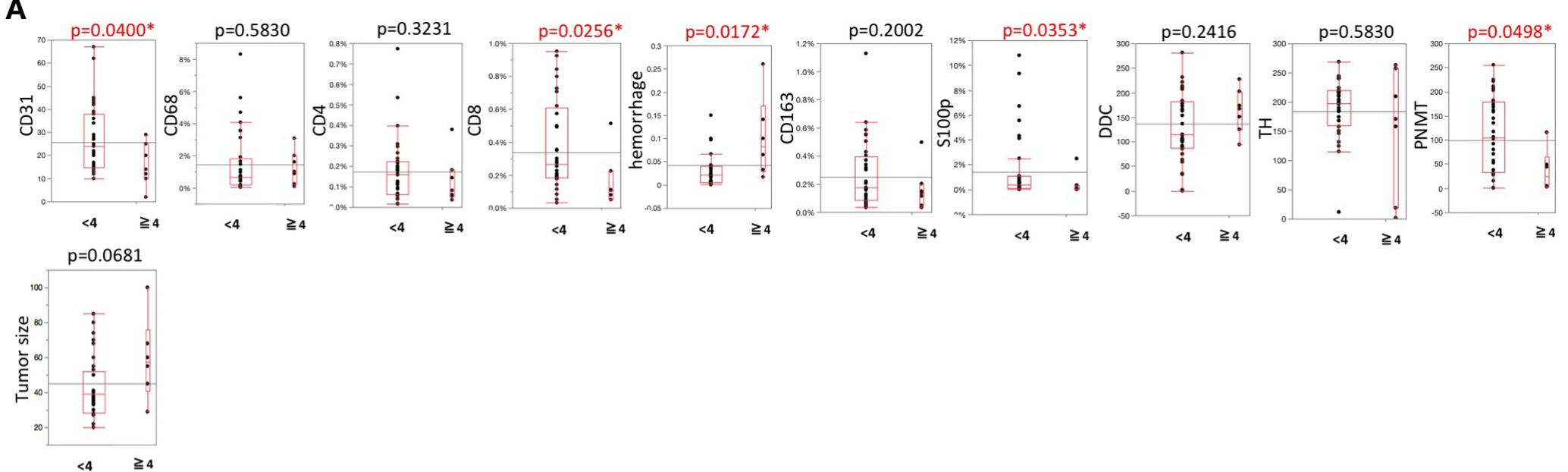

B
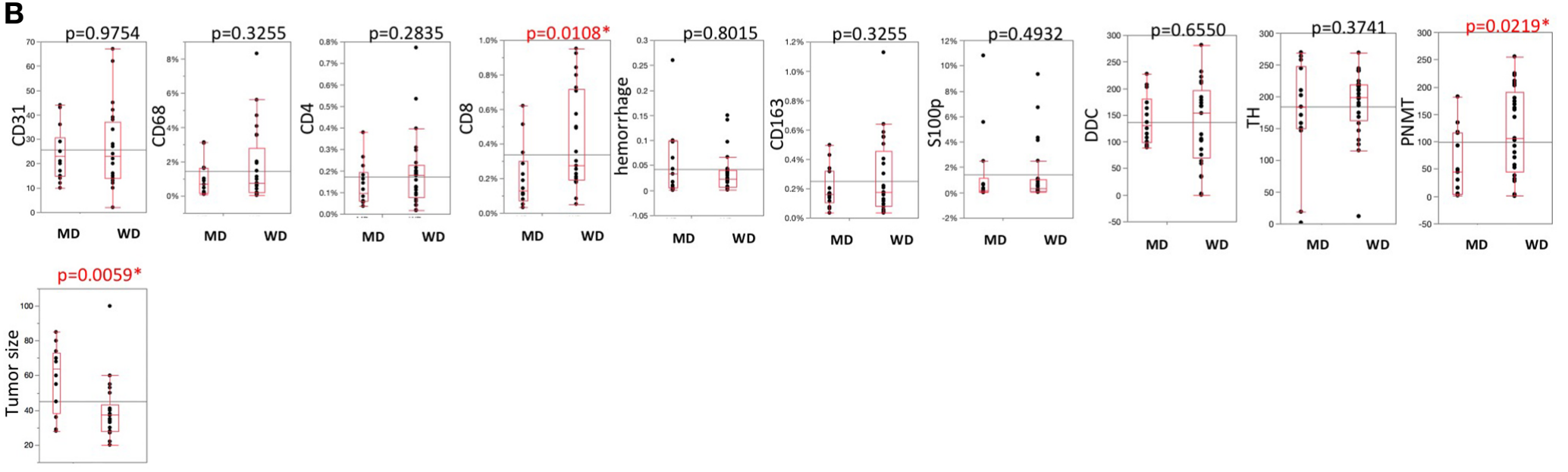

MD WD

FIGURE 3 | (A) Comparisons of TME-relevant markers, angiogenic marker, and catecholamine-synthesizing enzymes between PASS $<4$ and PASS $\geqq 4$. (B) Comparisons of TME-relevant markers, angiogenic

marker, and catecholamine-synthesizing enzymes between WD and MD according to the GAPP system. All of the comparisons are presented Figure 3. P value was on the head of each factor. 
ratio of intratumoral hemorrhage was significantly higher in PHEOs with PASS $\geqq 4$ than PASS $<4$ ( $\mathrm{p}=0.0172$ ).

\section{Comparison Between Well and Moderately Differentiated PHEOs According to GAPP Analysis}

Analysis based on GAPP scores revealed as follows: 26 welldifferentiated (WD), 13 moderately differentiated, and no poorly differentiated PEHOs (Figure 3). Both PNMT immunoreactivity $(\mathrm{p}=0.0219)$ and CD8 positive cells $(\mathrm{p}=0.0108)$ were significantly higher in WD- than MD-PHEOs. Tumor size was significantly smaller in WD- than MD-PHEOs $(p=0.0059)$. No other significant correlations were detected.

\section{Comparison of TME, Angiogenesis, and} Catecholamine-Synthesizing Enzymes With Results of PASS and GAPP Factors in Individual PHEOs

The histopathological factors of PASS and GAPP scoring systems were summarized in Table 3. Among individual factors of the PASS systems, the following were the numbers of positive individual cases per each factor: large nests of cells or diffuse growth in 10\% of tumor: six; necrosis: three; high cellularity: four; cellular monotony: two; presence of spindle shaped tumor cells: two; increased mitotic figure ( $>3$ per 10 high power fields): zero; atypical mitosis: one; extension of tumor into adjacent fat: three; vascular invasion: 13; capsular invasion: five cases; profound nuclear pleomorphism: five; nuclear hyperchromasia: three (Supplemental File 1).

Among the six GAPP factors examined in 39 PHEOs in our present study, 13 demonstrated abnormal histological features, 11 low cellularity (<150/HPF), 19 moderate cellularity (150-250/HPF) and nine high cellularity (>250/HPF), one comedo-necrosis, 13 capsule or vascular invasion, 26 low Ki-67 LI (<1\%), nine moderate Ki-67 LI (1$3 \%)$ and four high Ki-67 LI (>3\%), 10 norepinephrine predominant secretion (Supplemental File 2). CD68 positive cells were significantly higher in PHEOs with normal histological patterns than those with abnormal patterns $(p=0.0370)$. DDC in tumor cells was significantly more abundant in PHEOs with capsule or vascular invasion than those without $(\mathrm{p}=0.0172)$. TH in tumor cells was significantly more abundant in PHEOs with normal histological patterns than PHEOs with abnormal patterns $(\mathrm{p}=0.0236)$.

\section{Correlations Among TME Relevant Markers,} Angiogenic Markers, and Catecholamine-

Synthesizing Enzymes

Results were summarized in Table 4. CD163 positive cells were significantly positively correlated with CD4 $(\mathrm{p}=0.0405)$ and CD8 ( $p=0.0032)$ positive cells in the tumor. The ratio of intratumoral hemorrhage areas was inversely correlated with CD31-positive vessels $(p=0.0785)$. CD8 positive cells were significantly inversely correlated with GAPP score ( $\mathrm{p}=$ $0.0018)$. CD68 positive cells were positively correlated with $\mathrm{TH}$ $(\mathrm{p}=0.0901)$ and DDC $(\mathrm{p}=0.0168)$ immunoreactivity in tumor cells. S100p positive cells were significantly inversely correlated with PASS $(\mathrm{p}=0.0205)$ and GAPP $(\mathrm{p}=0.0217)$ scores. Hemorrhage score was significantly positively correlated with PASS score $(\mathrm{p}=0.0059)$. DDC was positively correlated with
TABLE 3 | PASS and GAPP systems.

PASS factors
Large nests or diffuse growth (>10\% of tumor volume)
Necrosis
High cellularity
Cellular monotony
Presence of spindle-shamed tumor cells
Atypical mitotic figures
Greater than 3 mitotic figures
Extension of tumor into adjacent fat
Vascular invasion
Capsular invasion
Profound nuclear pleomorphism
Nuclear hyperchromasia

Total

GAPP factors

Histological pattern

Zellballen

Large and irregular cell nest

Pseudorosette

0

Cellularity:

Low (<150 cells/U)

Moderate (150-250 cells/U)

High (>250 cells/U)

Score

2

2

2

2

1

Comedo necrosis:

Absence

Presence

Vascular or capsular invasion:

Absence

Presence

Ki-67 labeling index:

$<1 \%$

$1-3 \%$

$>3 \%$

Catecholamine type:

Epinephrine type (E or $E+N E)$

0

Norepinephrine type (NE or NE + AD)

1

Non-functioning type

0

Total maximum score

10

U, number of tumor cells under $\times 400$ magnification; $E$, epinephrine; NE, norepinephrine.

PASS score ( $\mathrm{p}=0.0365)$ but PNMT was inversely significantly correlated with PASS $(\mathrm{p}=0.0632)$ and GAPP score $(\mathrm{p}=0.0003)$.

\section{Correlations of TME Relevant Markers, Angiogenic Markers With Clinicopathological Factors in PHEOs}

The correlations among TME relevant markers, angiogenic markers and catecholamine-synthesizing enzymes and clinicopathological factors were summarized in Table 5. 
TABLE 4 | Correlations among TME-relevant, angiogenetic markers, and catecholamine-synthesizing enzymes.

\begin{tabular}{|c|c|c|c|c|c|c|c|c|c|c|c|c|}
\hline & CD31 & CD4 & CD8 & CD68 & CD163 & S100 & Ki-67 & TH & DDC & PNMT & PASS score & GAPP score \\
\hline CD31 & & & & & & & & $\begin{array}{l}\rho=0.0013 \\
p=0.9938\end{array}$ & $\begin{array}{l}\rho=-0.0277 \\
\rho=0.8688\end{array}$ & $\begin{array}{l}\rho=-0.0737 \\
p=0.6647\end{array}$ & $\begin{array}{l}\rho=-0.1980 \\
p=0.2333\end{array}$ & $\begin{array}{l}\rho=-0.0119 \\
p=0.9436\end{array}$ \\
\hline CD4 & $\begin{array}{l}\rho=0.1069 \\
p=0.5230\end{array}$ & & & & & & & $\begin{array}{l}\rho=-0.1340 \\
\rho=0.4160\end{array}$ & $\begin{array}{l}\rho=-0.1138 \\
p=0.4905\end{array}$ & $\begin{array}{l}\rho=0.2804 \\
p=0.0881\end{array}$ & $\begin{array}{l}\rho=-0.1894 \\
\rho=0.2482\end{array}$ & $\begin{array}{l}\rho=-0.1110 \\
\rho=0.5013\end{array}$ \\
\hline CD8 & $\begin{array}{l}\rho=-0.0650 \\
\rho=0.6982\end{array}$ & $\begin{array}{l}\rho=0.0395 \\
p=0.8114\end{array}$ & & & & & & $\begin{array}{l}\rho=-0.0312 \\
\rho=0.8505\end{array}$ & $\begin{array}{l}\rho=-0.1795 \\
\rho=0.2743\end{array}$ & $\begin{array}{l}\rho=0.1672 \\
p=0.3156\end{array}$ & $\begin{array}{l}\rho=-0.1714 \\
\rho=0.2969\end{array}$ & $\begin{array}{l}\rho=-0.4839 \\
p=0.0018^{*}\end{array}$ \\
\hline CD68 & $\begin{array}{l}\rho=-0.0629 \\
\rho=0.7077\end{array}$ & $\begin{array}{l}\rho=0.0759 \\
p=0.6460\end{array}$ & $\begin{array}{l}\rho=0.1822 \\
p=0.2669\end{array}$ & & & & & $\begin{array}{l}\rho=0.2751 \\
p=0.0901\end{array}$ & $\begin{array}{l}\rho=0.3806 \\
p=0.0168^{\star}\end{array}$ & $\begin{array}{l}\rho=0.2041 \\
p=0.2191\end{array}$ & $\begin{array}{l}\rho=0.0612 \\
p=0.7113\end{array}$ & $\begin{array}{l}\rho=-0.1848 \\
p=0.2600\end{array}$ \\
\hline CD163 & $\begin{array}{l}\rho=0.0460 \\
p=0.7839\end{array}$ & $\begin{array}{l}\rho=0.3296 \\
\rho=0.0405^{\star}\end{array}$ & $\begin{array}{l}\rho=0.4604 \\
\rho=0.0032^{*}\end{array}$ & $\begin{array}{l}\rho=-0.0200 \\
p=0.9036\end{array}$ & & & & $\begin{array}{l}\rho=0.0435 \\
p=0.7925\end{array}$ & $\begin{array}{l}\rho=0.0022 \\
p=0.9893\end{array}$ & $\begin{array}{l}\rho=0.0058 \\
p=0.9724\end{array}$ & $\begin{array}{l}\rho=-0.1593 \\
p=0.3327\end{array}$ & $\begin{array}{l}\rho=-0.1668 \\
p=0.3103\end{array}$ \\
\hline S100p & $\begin{array}{l}\rho=0.1404 \\
p=0.4005\end{array}$ & $\begin{array}{l}\rho=0.2008 \\
p=0.2203\end{array}$ & $\begin{array}{l}\rho=0.2642 \\
p=0.1041\end{array}$ & $\begin{array}{l}\rho=0.0277 \\
p=0.8669\end{array}$ & $\begin{array}{l}\rho=0.1054 \\
p=0.5232\end{array}$ & & & $\begin{array}{l}\rho=-0.0108 \\
\rho=0.9478\end{array}$ & $\begin{array}{l}\rho=-0.0420 \\
p=0.7996\end{array}$ & $\begin{array}{l}\rho=0.2185 \\
p=0.1875\end{array}$ & $\begin{array}{l}\rho=-0.3699 \\
p=0.0205^{\star}\end{array}$ & $\begin{array}{l}\rho=-0.3665 \\
\rho=0.0217^{\star}\end{array}$ \\
\hline Ki-67 & $\begin{array}{l}\rho=-0.0841 \\
p=0.6206\end{array}$ & $\begin{array}{l}\rho=0.1014 \\
p=0.5445\end{array}$ & $\begin{array}{l}\rho=0.0039 \\
p=0.9813\end{array}$ & $\begin{array}{l}\rho=0.0863 \\
p=0.6063\end{array}$ & $\begin{array}{l}\rho=0.1623 \\
p=0.3304\end{array}$ & $\begin{array}{l}\rho=-0.2206 \\
p=0.1832\end{array}$ & & $\begin{array}{l}\rho=0.1977 \\
p=0.2341\end{array}$ & $\begin{array}{l}\rho=-0.0012 \\
p=0.9943\end{array}$ & $\begin{array}{l}\rho=0.0028 \\
p=0.9867\end{array}$ & $\begin{array}{l}\rho=0.2319 \\
p=0.1612\end{array}$ & $\begin{array}{l}\rho=0.2909 \\
p=0.0764\end{array}$ \\
\hline Hemorrhage & $\begin{array}{l}\rho=-0.2955 \\
\rho=0.0758\end{array}$ & $\begin{array}{l}\rho=-0.1605 \\
\rho=0.3358\end{array}$ & $\begin{array}{l}\rho=0.0630 \\
p=0.7070\end{array}$ & $\begin{array}{l}\rho=0.0667 \\
p=0.6909\end{array}$ & $\begin{array}{l}\rho=0.0454 \\
\rho=0.7865\end{array}$ & $\begin{array}{l}\rho=-0.1501 \\
\rho=0.3684\end{array}$ & $\begin{array}{l}\rho=-0.1555 \\
\rho=0.3581\end{array}$ & $\begin{array}{l}\rho=0.0055 \\
p=0.9740\end{array}$ & $\begin{array}{l}\rho=0.1228 \\
p=0.4625\end{array}$ & $\begin{array}{l}\rho=-0.0125 \\
\rho=0.9417\end{array}$ & $\begin{array}{l}\rho=0.4385 \\
\rho=0.0059^{\star}\end{array}$ & $\begin{array}{l}\rho=0.0575 \\
\rho=0.7317\end{array}$ \\
\hline PASS score & & & & & & & & $\begin{array}{l}\rho=-0.1492 \\
\rho=0.3648\end{array}$ & $\begin{array}{l}\rho=0.3376 \\
p=0.0356^{\star}\end{array}$ & $\begin{array}{l}\rho=-0.3044 \\
\rho=0.0632\end{array}$ & & \\
\hline GAPP score & & & & & & & & $\begin{array}{l}\rho=-0.1598 \\
p=0.3313\end{array}$ & $\begin{array}{l}\rho=0.1808 \\
p=0.2708\end{array}$ & $\begin{array}{l}\rho=-0.5580 \\
p=0.0003^{*}\end{array}$ & & \\
\hline
\end{tabular}

${ }^{*} p<0.05$ 
TABLE 5 | Correlations among TME-relevant markers, plasma, and urine catecholamines and clinicopathological factors.

\begin{tabular}{|c|c|c|c|c|c|}
\hline & Plasma AD & Plasma NAD & Urine AD & Urine NAD & Tumor size \\
\hline \multirow[t]{2}{*}{ CD31 } & $\rho=0.0415$ & $\rho=0.1527$ & $\rho=-0.0875$ & $\rho=0.1179$ & $\rho=-0.0226$ \\
\hline & $p=0.8075$ & $p=0.3668$ & $p=0.6119$ & $p=0.4935$ & $p=0.8957$ \\
\hline \multirow[t]{2}{*}{ CD4 } & $\rho=-0.0677$ & $\rho=-0.0748$ & $\rho=0.0937$ & $\rho=0.0254$ & $\rho=-0.0235$ \\
\hline & $p=0.6863$ & $p=0.6556$ & $p=0.5814$ & $p=0.8815$ & $p=0.8901$ \\
\hline \multirow[t]{2}{*}{ CD8 } & $\rho=0.3175$ & $\rho=-0.0718$ & $\rho=0.2197$ & $\rho=-0.1190$ & $\rho=-0.3665$ \\
\hline & $p=0.0521$ & $p=0.6683$ & $p=0.1914$ & $p=0.4829$ & $p=0.0257^{*}$ \\
\hline \multirow[t]{2}{*}{ CD68 } & $\rho=0.2738$ & $\rho=0.1993$ & $\rho=0.1496$ & $\rho=-0.1107$ & $\rho=-0.3305$ \\
\hline & $p=0.0962$ & $p=0.2303$ & $p=0.3768$ & $p=0.5142$ & $p=0.0457^{*}$ \\
\hline \multirow[t]{2}{*}{ CD163 } & $\rho=0.0359$ & $\rho=0.0638$ & $\rho=-0.0211$ & $\rho=0.0318$ & $\rho=0.0638$ \\
\hline & $p=0.8304$ & $p=0.7035$ & $p=0.9013$ & $p=0.8519$ & $p=0.7077$ \\
\hline \multirow[t]{2}{*}{ S100p } & $\rho=0.0141$ & $\rho=0.0464$ & $\rho=0.0598$ & $\rho=0.0537$ & $\rho=-0.4676$ \\
\hline & $p=0.9331$ & $p=0.7820$ & $p=0.7254$ & $p=0.7523$ & $p=0.0035^{*}$ \\
\hline \multirow[t]{2}{*}{ Ki-67 } & $\rho=-0.0145$ & $\rho=-0.1544$ & $\rho=0.0049$ & $\rho=-0.2530$ & $\rho=-0.0181$ \\
\hline & $p=0.9322$ & $p=0.3616$ & $p=0.9774$ & $p=0.1365$ & $p=0.9168$ \\
\hline \multirow[t]{2}{*}{ Hemorrhage } & $\rho=0.0425$ & $\rho=-0.2718$ & $\rho=0.0644$ & $\rho=-0.2752$ & $\rho=0.0595$ \\
\hline & $p=0.8028$ & $p=0.1037$ & $p=0.7091$ & $p=0.1043$ & $p=0.7301$ \\
\hline \multirow[t]{2}{*}{ TH } & $\rho=-0.0956$ & $\rho=0.1085$ & $\rho=-0.2388$ & $\rho=-0.1183$ & $\rho=-0.3171$ \\
\hline & $p=0.5679$ & $p=0.5169$ & $p=0.1547$ & $p=0.4856$ & $p=0.0558$ \\
\hline \multirow[t]{2}{*}{ DDC } & $\rho=-0.1402$ & $\rho=0.1288$ & $\rho=-0.1721$ & $\rho=0.0782$ & $\rho=-0.1125$ \\
\hline & $p=0.4010$ & $p=0.4408$ & $p=0.3083$ & $p=0.6453$ & $p=0.5075$ \\
\hline \multirow[t]{2}{*}{ PNMT } & $\rho=0.2564$ & $\rho=-0.2727$ & $\rho=0.3052$ & $\rho=-0.3586$ & $\rho=-0.5290$ \\
\hline & $p=0.1256$ & $p=0.1025$ & $p=0.0703$ & $p=0.0318^{*}$ & $p=0.0009^{*}$ \\
\hline \multirow[t]{2}{*}{ PASS score } & $\rho=-0.0269$ & $\rho=-0.1252$ & $\rho=-0.0056$ & $\rho=-0.1274$ & $\rho=0.2961$ \\
\hline & $p=0.8726$ & $p=0.4539$ & $p=0.9739$ & $p=0.4523$ & $p=0.0710$ \\
\hline \multirow[t]{2}{*}{ GAPP score } & $\rho=-0.3787$ & $\rho=0.0396$ & $\rho=-0.3115$ & $\rho=0.0977$ & $\rho=0.5316$ \\
\hline & $\mathrm{p}=0.0191^{\star}$ & $p=0.8136$ & $p=0.0605$ & $p=0.5653$ & $p=0.0006^{*}$ \\
\hline
\end{tabular}

$*: p<0.05$.

Plasma adrenaline levels were significantly positively correlated with CD8 ( $\mathrm{p}=0.0521)$ and CD68 ( $\mathrm{p}=0.0962)$ positive cells and significantly inversely correlated with GAPP score $(p=0.0191)$. Urinary AD was inversely correlated with GAPP score ( $\mathrm{p}=$ 0.0605). PNMT expression was significantly inversely correlated with urinary NAD ( $\mathrm{p}=0.0318)$. Tumor size was significantly inversely correlate with CD8 ( $\mathrm{p}=0.0257)$, CD68 ( $\mathrm{p}=0.0457)$, S100 ( $=0.0035)$, PNMT $(p=0.0009)$ and positively correlated with PASS $(\mathrm{p}=0.0710)$ and GAPP score $(\mathrm{p}=0.0006)$.

\section{DISCUSSION}

TME including tumor infiltrating lymphocytes (TILs), tumorassociated macrophages (TAMs) as well as angiogenesis has all been reported to play pivotal roles in not only clinical but also therapeutic outcomes in various human malignancies. However, TME and other relevant phenomena have not necessarily been well studied in adrenal medullary tumor including PHEOs although decreased S100 was detected in benign PHEOs (17, $18,30)$. In our present study, we did compare the status of S100 positive cells with results of PASS analysis and obtained similar findings reported above (7). Both PASS and GAPP scoring systems have been used relatively widely for surgical pathologists to evaluate the malignant potential of the patients with PHEOs. PASS score could be obtained only from routinely available histopathological findings and tumor with PASS of four or more than four score was considered malignant (7). However, relatively marked inter- and intra-observer variations have been reported in application of PASS scoring system (31). Therefore, we also employed GAPP system as well, which included not only morphological features but also catecholamine secretary phenotypes and Ki-67 labeling index (8), in order to further explore the malignant potential of PHEOs in a more precise fashion (32).

In this study, we firstly evaluated the details of TILs. The status of $\mathrm{CD}^{+}$TILs has been generally reported to be correlated with better prognosis (33-35). Our results did demonstrate that $\mathrm{CD}^{+}$TILs could play pivotal roles in possible biological behavior of PHEOs and its abundance could also represent relatively benign tumors as reported in other human malignancies (33-35). However, it awaits further investigations for clarification.

Dendritic cells activated anti-tumor $\mathrm{CD}^{+} \mathrm{T}$ lymphocytes (CTLs) by presenting tumor antigen and then effector CTLs executed its roles of eliminating tumor cells (36). CTLs can induce an apoptosis of tumor cell by FasL pathway and can also release some cytokines to induce cytotoxicity in tumor cells (37). In our present study, we demonstrated an inverse correlation between CTLs and tumor size, which indicated that CTLs could suppress the expansion of tumor. However, CTLs were also suppressed in malignant PHEOs possibly by excessive catecholamine derived from tumor cells, themselves (38). Catecholamine was reported to suppress $\mathrm{T}$ lymphocyte such as T-helper 1/2, cytotoxic CD8 ${ }^{+}$T-lymphocytes and NK cells (39$41)$. Both epinephrine and norepinephrine were also reported to 
exert inhibitory effects on T-cell proliferation due to chronic mild stress in mice (42). Therefore, results of our present study also indicated that CTLs could be suppressed by excessive catecholamine produced by malignant PHEOs. In addition, in our present study, $\mathrm{CD}^{+} 8^{+}$cells were significantly higher in PHEOs with normal histological patterns and negatively correlated with tumor size. Intratumoral CD163 positive cells were significantly positively correlated with the status of intratumoral CD4 and CD8 infiltrating cells. Macrophages constituted the great majority of cellular components in tumor stroma with noticeable plasticity and could be further subclassified into M1 and M2 phenotypes (37). M1 macrophages were classically defined as activated macrophages and reported to play pro-inflammation roles resulting in overall antitumor effects $(37,43,44)$. On the other hand, M2 macrophages were reported to have opposite roles toward M1 macrophages resulting in pro-tumor effects $(37,44,45)$. In addition, the differentiation to M1 or M2 macrophages was reported to be dependent on cytokines (44). For instance, differentiation to M1 macrophages could be induced by IFN- $\gamma$, TNF- $\alpha$, IL-8, and IL-12 secreted from CTLs. M2 macrophages were reported to be induced by STAT3, IL4, and IL-10 and to subsequently suppress $\mathrm{T}$ cell function. In addition, M2 macrophages were the representative subtypes of macrophages in TME of most human malignancies including breast, urinary bladder, and prostate carcinoma $(46,47)$. Results of our present study did demonstrate that TAMs in PHEOs could be predominantly reprogrammed into $\mathrm{CD} 68^{+} \mathrm{M} 1$ rather than CD163+M2 macrophages, which exerted potential antitumoral effects by secreting different cytokines (44, 48). However further investigations are required to clarify the details.

Recently, TILs have been also reported to be associated with overall survival and recurrence-free survival of the patients with adrenocortical carcinomas (ACCs). However, of particular interest, the difference between ACCs and PHEOs was that not only $\mathrm{CD}^{+}$but also $\mathrm{CD}^{+} \mathrm{T}$ lymphocytes were related to relatively better clinical outcome in the patients with ACCs (15). $\mathrm{CD}^{+} \mathrm{T}$ cells are helper $\mathrm{T}$ cells, stimulating CTLs activities (37). In addition, ACCs were also relatively frequently detected in childhood and increased TILs were reported in those young ACCs patients than adults (49). The most notable difference between ACCs and PHEOs was considered the effects of different hormones on TILs. The great majority of PHEOs were characterized by catecholamine excess, which could suppress T-cell activity. On the other hand, ACC was characterized by the excessive steroids including relatively abundant precursor steroids and steroid hormones could in general hamper anti-tumor roles of T-cells (50-53). Therefore, tumor microenvironment could be influenced by those hormones secreted by tumor cells, but it awaits further investigations for clarification.

Angiogenesis is indispensable and pivotal for tumor growth, invasion and metastasis (54). Hypoxia has been reported one of the most pivotal causes of angiogenesis in PHEOs (55). Increased endothelial growth factor (VEGF) has been reported in malignant PHEOs and to be associated with hypoxia inducible factor (HIF) (56-59). PHEOs have been well known to be associated with increased angiogenesis due to pseudohypoxic signaling pathway (60). Among the factors involved in this pathway, SDHXs were the most susceptive genes (60). Pseudohypoxic subtype has been also reported to be associated with aggressive biological behavior (60). The pathophysiological features of this particular phenotype could therefore promote the neoplastic angiogenesis. In our present study, intratumoral hemorrhage was abundantly detected in histologically highgraded tumors, which could also result in relatively low CD31 status in histologically low-graded tumors. However, genetic testing was not performed as a routine in our institution and this could also represent the limitation of our present study.

In general, norepinephrine-producing PHEOs were reported to accompany PNMT deficiency which is considered to be less differentiated than epinephrine-producing PHEOs $(7,17)$. Dopamine-secreting PHEOs were also considered less differentiated or immature and had a high prevalence of malignancy $(59,60)$. Results of our present study were also consistent with those findings above. The absence of TH in some PHEOs in our present study could not only result in nonfunctional nature of the tumors but also in increased aggressive biological behavior $(61,62)$. Therefore, the abnormal elevation of plasma dopamine or the absence of $\mathrm{TH}$ immunoreactivity in PHEOs could indicate more aggressive biological behavior of the tumor.

It is true that there are several limitations in our present study. The number of the cases examined was relatively small. In addition, the cases examined were relatively new cases which could make it difficult to obtain the long-term clinical outcome of the patients. Genetic testing was not performed routinely in our hospital. Therefore, those above were considered limitations of this study. Further investigations are also required to clarify the detailed mechanisms of interaction between TME and tumor cells in PHEOs.

In summary, we firstly presented detailed features of TILs and TAMs in PHEOs. CD8 and CD68 could also serve as biomarkers of well-differentiated or histologically low-scored PHEOs. Benign PHEOs harbored smaller sized tumor than malignant ones, also related both TILs and TAMs. Poorly differentiated PHEOs had increased incidence of intratumoral hemorrhage and the absence of $\mathrm{TH}$ in tumor cells.

\section{DATA AVAILABILITY STATEMENT}

The raw data supporting the conclusions of this article will be made available by the authors, without undue reservation.

\section{ETHICS STATEMENT}

The studies involving human participants were reviewed and approved by the Institutional Review Board of Tohoku 
University School of Medicine (2018-1-669). The patients/ participants provided their written informed consent to participate in this study.

\section{AUTHOR CONTRIBUTIONS}

$\mathrm{XG}, \mathrm{YY}$, and AP contributed to staining and evaluation of $\mathrm{H} \& \mathrm{E}$ and IHC sections. YT, KO, YO, and RM contributed to the collection of clinical data from Tohoku University hospital. YN, FS, and HS supervised all of the present study. All authors contributed to the article and approved the submitted version.

\section{REFERENCES}

1. Farrugia FA, Charalampopoulos A. Pheochromocytoma. Endocr Regul (2019) 53:191-212. doi: 10.2478/enr-2019-0020

2. Farrugia FA, Martikos G, Tzanetis P, Charalampopoulos A, Misiakos E, Zavras N, et al. Pheochromocytoma, diagnosis and treatment: Review of the literature. Endocr Regul (2017) 51:168-81. doi: 10.1515/enr-2017-0018

3. Farrugia FA, Georgios M, Panagiotis T, Nikolaos Z, Anestis C, Dimitrios S, et al. Adrenal incidentaloma or epinephroma and review of the literature. Differential diagnosis of adrenal incidentaloma. Khirurgiia (Sofiia) (2016) 82:120-8.

4. Lam AK. Update on Adrenal Tumours in 2017 World Health Organization (WHO) of Endocrine Tumours. Endocr Pathol (2017) 28:213-27. doi: 10.1007/s12022-017-9484-5

5. Bravo EL. Pheochromocytoma: new concepts and future trends. Kidney Int (1991) 40:544-56. doi: 10.1038/ki.1991.244

6. Lloyd RVOR, Kloppel G, Rosai J. WHO classification of tumours: pathology and genetics of tumours of endocrine organs. (2017).

7. Thompson LD. Pheochromocytoma of the Adrenal gland Scaled Score (PASS) to separate benign from malignant neoplasms: a clinicopathologic and immunophenotypic study of 100 cases. Am J Surg Pathol (2002) 26:55166. doi: 10.1097/00000478-200205000-00002

8. Kimura N, Takayanagi R, Takizawa N, Itagaki E, Katabami T, Kakoi N, et al. Pathological grading for predicting metastasis in phaeochromocytoma and paraganglioma. Endocr Relat Cancer (2014) 21:405-14. doi: 10.1530/ERC-130494

9. Chew V, Toh HC, Abastado JP. Immune microenvironment in tumor progression: characteristics and challenges for therapy. J Oncol (2012) 2012:608406. doi: 10.1155/2012/608406

10. Quail DF, Joyce JA. Microenvironmental regulation of tumor progression and metastasis. Nat Med (2013) 19:1423-37. doi: 10.1038/nm.3394

11. Hanahan D, Coussens LM. Accessories to the crime: functions of cells recruited to the tumor microenvironment. Cancer Cell (2012) 21:309-22. doi: 10.1016/j.ccr.2012.02.022

12. Joyce JA, Fearon DT. T cell exclusion, immune privilege, and the tumor microenvironment. Science (2015) 348:74-80. doi: 10.1126/science.aaa6204

13. Rahat MA, Coffelt SB, Granot Z, Muthana M, Amedei A. Macrophages and Neutrophils: Regulation of the Inflammatory Microenvironment in Autoimmunity and Cancer. Mediators Inflamm (2016) 2016:5894347. doi: 10.1155/2016/5894347

14. Kitawaki Y, Nakamura Y, Kubota-Nakayama F, Yamazaki Y, Miki Y, Hata S, et al. Tumor microenvironment in functional adrenocortical adenomas: immune cell infiltration in cortisol-producing adrenocortical adenoma. Hum Pathol (2018) 77:88-97. doi: 10.1016/j.humpath.2018.03.016

15. Landwehr LS, Altieri B, Schreiner J, Sbiera I, Weigand I, Kroiss M, et al. Interplay between glucocorticoids and tumor-infiltrating lymphocytes on the prognosis of adrenocortical carcinoma. J Immunother Cancer (2020) 8. doi: 10.1136/jitc-2019-000469

16. Liu Q, Djuricin G, Staren ED, Gattuso P, Gould VE, Shen J, et al. Tumor angiogenesis in pheochromocytomas and paragangliomas. Surgery (1996) 120:938-42; discussion 942-933. doi: 10.1016/s0039-6060(96)80037-7

\section{FUNDING}

XG was supported by Global Hagi scholarship by Tohoku University. This work was supported by a Health Labour Sciences Research Grant (No. H29-Nanji-Ippan-046).

\section{SUPPLEMENTARY MATERIAL}

The Supplementary Material for this article can be found online at: https://www.frontiersin.org/articles/10.3389/fendo.2020. 587779/full\#supplementary-material

17. Kulkarni MM, Khandeparkar SG, Deshmukh SD, Karekar RR, Gaopande VL, Joshi AR, et al. Risk Stratification in Paragangliomas with PASS (Pheochromocytoma of the Adrenal Gland Scaled Score) and Immunohistochemical Markers. J Clin Diagn Res (2016) 10:EC01-4. doi: 10.7860/JCDR/2016/20565.8419

18. Konosu-Fukaya S, Omata K, Tezuka Y, Ono Y, Aoyama Y, Satoh F, et al. Catecholamine-Synthesizing Enzymes in Pheochromocytoma and Extraadrenal Paraganglioma. Endocr Pathol (2018) 29:302-9. doi: 10.1007/s12022-018-9544-5

19. Chan MS, Chen SF, Felizola SJ, Wang L, Nemoto N, Tamaki K, et al. Correlation of tumor-infiltrative lymphocyte subtypes alteration with neoangiogenesis before and after neoadjuvant chemotherapy treatment in breast cancer patients. Int J Biol Markers (2014) 29:e193-203. doi: 10.5301/ jbm.5000082

20. Konno-Kumagai T, Fujishima F, Nakamura Y, Nakano T, Nagai T, Kamei T, et al. Programmed death-1 ligands and tumor infiltrating $\mathrm{T}$ lymphocytes in primary and lymph node metastasis of esophageal cancer patients. Dis Esophagus (2019) 32. doi: 10.1093/dote/doy063

21. Chan MS, Wang L, Chanplakorn N, Tamaki K, Ueno T, Toi M, et al. Effects of estrogen depletion on angiogenesis in estrogen-receptor-positive breast carcinoma-an immunohistochemical study of vasohibin-1 and CD31 with correlation to pathobiological response of the patients in neoadjuvant aromatase inhibitor therapy. Expert Opin Ther Targets (2012) 16 Suppl 1: S69-78. doi: 10.1517/14728222.2011.628938

22. Yazdani S, Kasajima A, Tamaki K, Nakamura Y, Fujishima F, Ohtsuka H, et al. Angiogenesis and vascular maturation in neuroendocrine tumors. Hum Pathol (2014) 45:866-74. doi: 10.1016/j.humpath.2013.09.024

23. Oka N, Kasajima A, Konukiewitz B, Sakurada A, Okada Y, Kameya T, et al. Classification and Prognostic Stratification of Bronchopulmonary Neuroendocrine Neoplasms. Neuroendocrinology (2020) 110:393-403. doi: 10.1159/000502776

24. Yazdani S, Miki Y, Tamaki K, Ono K, Iwabuchi E, Abe K, et al. Proliferation and maturation of intratumoral blood vessels in non-small cell lung cancer. Hum Pathol (2013) 44:1586-96. doi: 10.1016/j.humpath.2013.01.004

25. Yamazaki Y, Omata K, Tezuka Y, Ono Y, Morimoto R, Adachi Y, et al. Tumor Cell Subtypes Based on the Intracellular Hormonal Activity in KCNJ5Mutated Aldosterone-Producing Adenoma. Hypertension (2018) 72:632-40. doi: 10.1161/HYPERTENSIONAHA.118.10907

26. Gao X, Yamazaki Y, Tezuka Y, Onodera Y, Ogata H, Omata K, et al. The crosstalk between aldosterone and calcium metabolism in primary aldosteronism: A possible calcium metabolism-associated aberrant "neoplastic" steroidogenesis in adrenals. J Steroid Biochem Mol Biol (2019) 193:105434. doi: 10.1016/j.jsbmb.2019.105434

27. Konosu-Fukaya S, Nakamura Y, Satoh F, Felizola SJ, Maekawa T, Ono Y, et al. 3beta-Hydroxysteroid dehydrogenase isoforms in human aldosteroneproducing adenoma. Mol Cell Endocrinol (2015) 408:205-12. doi: 10.1016/ j.mce.2014.10.008

28. McCarty KSJr., Miller LS, Cox EB, Konrath J, McCarty KSSr. Estrogen receptor analyses. Correlation of biochemical and immunohistochemical methods using monoclonal antireceptor antibodies. Arch Pathol Lab Med (1985) 109:716-21.

29. Yamazaki Y, Nakamura Y, Omata K, Ise K, Tezuka Y, Ono Y, et al. Histopathological Classification of Cross-Sectional Image-Negative 
Hyperaldosteronism. J Clin Endocrinol Metab (2017) 102:1182-92. doi: 10.1210/jc.2016-2986

30. Farhat NA, Powers JF, Shepard-Barry A, Dahia P, Pacak K, Tischler AS. A Previously Unrecognized Monocytic Component of Pheochromocytoma and Paraganglioma. Endocr Pathol (2019) 30:90-5. doi: 10.1007/s12022-019-9575-6

31. Wu D, Tischler AS, Lloyd RV, DeLellis RA, de Krijger R, van Nederveen F, et al. Observer variation in the application of the Pheochromocytoma of the Adrenal Gland Scaled Score. Am J Surg Pathol (2009) 33:599-608. doi: 10.1097/PAS.0b013e318190d12e

32. Fishbein L. Pheochromocytoma and Paraganglioma: Genetics, Diagnosis, and Treatment. Hematol Oncol Clin North Am (2016) 30:135-50. doi: 10.1016/ j.hoc.2015.09.006

33. Troiano G, Caponio VCA, Adipietro I, Tepedino M, Santoro R, Laino L, et al. Prognostic significance of CD68(+) and CD163(+) tumor associated macrophages in head and neck squamous cell carcinoma: A systematic review and meta-analysis. Oral Oncol (2019) 93:66-75. doi: 10.1016/ j.oraloncology.2019.04.019

34. Ni YH, Ding L, Huang XF, Dong YC, Hu QG, Hou YY. Microlocalization of CD68+ tumor-associated macrophages in tumor stroma correlated with poor clinical outcomes in oral squamous cell carcinoma patients. Tumour Biol (2015) 36:5291-8. doi: 10.1007/s13277-015-3189-5

35. Lin Z, Gu J, Cui X, Huang L, Li S, Feng J, et al. Deciphering Microenvironment of NSCLC based on CD8+ TIL Density and PD-1/PD-L1 Expression. J Cancer (2019) 10:211-22. doi: 10.7150/jca.26444

36. Nouri-Shirazi M, Banchereau J, Bell D, Burkeholder S, Kraus ET, Davoust J, et al. Dendritic cells capture killed tumor cells and present their antigens to elicit tumor-specific immune responses. J Immunol (2000) 165:3797-803. doi: 10.4049/jimmunol.165.7.3797

37. Farhood B, Najafi M, Mortezaee K. CD8(+) cytotoxic T lymphocytes in cancer immunotherapy: A review. J Cell Physiol (2019) 234:8509-21. doi: 10.1002/ jcp. 27782

38. Sarkar C, Chakroborty D, Basu S. Neurotransmitters as regulators of tumor angiogenesis and immunity: the role of catecholamines. J Neuroimmune Pharmacol (2013) 8:7-14. doi: 10.1007/s11481-012-9395-7

39. Madden KS, Sanders VM, Felten DL. Catecholamine influences and sympathetic neural modulation of immune responsiveness. Annu Rev Pharmacol Toxicol (1995) 35:417-48. doi: 10.1146/annurev.pa.35.040195.002221

40. Nasi G, Ahmed T, Rasini E, Fenoglio D, Marino F, Filaci G, et al. Dopamine inhibits human CD8+ Treg function through D1-like dopaminergic receptors. J Neuroimmunol (2019) 332:233-41. doi: 10.1016/j.jneuroim.2019.02.007

41. Inbar S, Neeman E, Avraham R, Benish M, Rosenne E, Ben-Eliyahu S. Do stress responses promote leukemia progression? An animal study suggesting a role for epinephrine and prostaglandin-E2 through reduced NK activity. PloS One (2011) 6:e19246. doi: 10.1371/journal.pone.0019246

42. Edgar VA, Silberman DM, Cremaschi GA, Zieher LM, Genaro AM. Altered lymphocyte catecholamine reactivity in mice subjected to chronic mild stress. Biochem Pharmacol (2003) 65:15-23. doi: 10.1016/s0006-2952(02)01457-0

43. Parayath NN, Parikh A, Amiji MM. Repolarization of Tumor-Associated Macrophages in a Genetically Engineered Nonsmall Cell Lung Cancer Model by Intraperitoneal Administration of Hyaluronic Acid-Based Nanoparticles Encapsulating MicroRNA-125b. Nano Lett (2018) 18:3571-9. doi: 10.1021/ acs.nanolett.8b00689

44. Wang N, Liang $\mathrm{H}$, Zen K. Molecular mechanisms that influence the macrophage m1-m2 polarization balance. Front Immunol (2014) 5:614. doi: 10.3389/fimmu.2014.00614

45. Genard G, Wera AC, Huart C, Le Calve B, Penninckx S, Fattaccioli A, et al. Proton irradiation orchestrates macrophage reprogramming through NFkappaB signaling. Cell Death Dis (2018) 9:728. doi: 10.1038/s41419-018-0757-9

46. Mantovani A, Marchesi F, Malesci A, Laghi L, Allavena P. Tumour-associated macrophages as treatment targets in oncology. Nat Rev Clin Oncol (2017) 14:399-416. doi: 10.1038/nrclinonc.2016.217

47. Ruffell BCoussens LM. Macrophages and therapeutic resistance in cancer. Cancer Cell (2015) 27:462-72. doi: 10.1016/j.ccell.2015.02.015
48. Yamate J, Izawa T, Kuwamura M. Histopathological Analysis of Rat Hepatotoxicity Based on Macrophage Functions: in Particular, an Analysis for Thioacetamide-induced Hepatic Lesions. Food Saf (Tokyo) (2016) 4:6173. doi: 10.14252 /foodsafetyfscj. 2016012

49. Parise IZS, Parise GA, Noronha L, Surakhy M, Woiski TD, Silva DB, et al. The Prognostic Role of CD8(+) T Lymphocytes in Childhood Adrenocortical Carcinomas Compared to Ki-67, PD-1, PD-L1, and the Weiss Score. Cancers (Basel) (2019) 11. doi: 10.3390/cancers11111730

50. Trigunaite A, Dimo J, Jorgensen TN. Suppressive effects of androgens on the immune system. Cell Immunol (2015) 294:87-94. doi: 10.1016/ j.cellimm.2015.02.004

51. Rasmuson T, Ljungberg B, Grankvist K, Jacobsen J, Olsson T. Increased serum cortisol levels are associated with high tumour grade in patients with renal cell carcinoma. Acta Oncol (2001) 40:83-7. doi: 10.1080/028418601750071118

52. Taves MD, Hamden JE, Soma KK. Local glucocorticoid production in lymphoid organs of mice and birds: Functions in lymphocyte development. Horm Behav (2017) 88:4-14. doi: 10.1016/j.yhbeh.2016.10.022

53. Trenti A, Tedesco S, Boscaro C, Trevisi L, Bolego C, Cignarella A. Estrogen, Angiogenesis, Immunity and Cell Metabolism: Solving the Puzzle. Int J Mol Sci (2018) 19. doi: 10.3390/ijms19030859

54. Sasano H, Suzuki T. Pathological evaluation of angiogenesis in human tumor. BioMed Pharmacother (2005) 59 Suppl 2:S334-336. doi: 10.1016/s0753-3322 (05) 80068 - $\mathrm{x}$

55. Favier J, Gimenez-Roqueplo AP. Pheochromocytomas: the (pseudo)-hypoxia hypothesis. Best Pract Res Clin Endocrinol Metab (2010) 24:957-68. doi: 10.1016/j.beem.2010.10.004

56. Pollard PJ, El-Bahrawy M, Poulsom R, Elia G, Killick P, Kelly G, et al. Expression of HIF-1alpha, HIF-2alpha (EPAS1), and their target genes in paraganglioma and pheochromocytoma with VHL and SDH mutations. J Clin Endocrinol Metab (2006) 91:4593-8. doi: 10.1210/jc.2006-0920

57. Salmenkivi K, Heikkila P, Liu J, Haglund C, Arola J. VEGF in 105 pheochromocytomas: enhanced expression correlates with malignant outcome. APMIS (2003) 111:458-64. doi: 10.1034/j.1600-0463.2003.1110402.x

58. Zielke A, Middeke M, Hoffmann S, Colombo-Benkmann M, Barth P, Hassan I, et al. VEGF-mediated angiogenesis of human pheochromocytomas is associated to malignancy and inhibited by anti-VEGF antibodies in experimental tumors. Surgery (2002) 132:1056-63; discussion 1063. doi: $10.1067 / \mathrm{msy} .2002 .128613$

59. Feng F, Zhu Y, Wang X, Wu Y, Zhou W, Jin X, et al. Predictive factors for malignant pheochromocytoma: analysis of 136 patients. J Urol (2011) 185:1583-90. doi: 10.1016/j.juro.2010.12.050

60. Amorim-Pires D, Peixoto J, Lima J. Hypoxia Pathway Mutations in Pheochromocytomas and Paragangliomas. Cytogenet Genome Res (2016) 150:227-41. doi: 10.1159/000457479

61. Takahashi H, Nakashima S, Kumanishi T, kuta F. Paragangliomas of the craniocervical region. An immunohistochemical study on tyrosine hydroxylase. Acta Neuropathol (1987) 73:227-32. doi: 10.1007/BF00686615

62. Kimura N, Miura Y, Nagatsu I, Nagura H. Catecholamine synthesizing enzymes in 70 cases of functioning and non-functioning phaeochromocytoma and extraadrenal paraganglioma. Virchows Arch A Pathol Anat Histopathol (1992) 421:25-32. doi: 10.1007/BF01607135

Conflict of Interest: The authors declare that the research was conducted in the absence of any commercial or financial relationships that could be construed as a potential conflict of interest.

Copyright $\odot 2020$ Gao, Yamazaki, Pecori, Tezuka, Ono, Omata, Morimoto, Nakamura Satoh and Sasano. This is an open-access article distributed under the terms of the Creative Commons Attribution License (CC BY). The use, distribution or reproduction in other forums is permitted, provided the original author(s) and the copyright owner(s) are credited and that the original publication in this journal is cited, in accordance with accepted academic practice. No use, distribution or reproduction is permitted which does not comply with these terms. 